$\underline{\text { Supporting information }}$

\title{
Photoinduced Charge Transfer in Donor-Bridge-Acceptor in One- and Two-photon Absorption: Sequential and Super-exchange Mechanisms
}

\author{
Xijiao Mu, ${ }^{1,+}$ Xinxin Wang, ${ }^{1, *}$ Jun Quan, ${ }^{2, *}$ Mengtao Sun*
}

1. School of Mathematics and Physics, Center for Green Innovation, Advanced Innovation Center for Materials Genome Engineering, Beijing Key Laboratory for Magneto-Photoelectrical Composite and Interface Science, University of Science and Technology, Beijing, Beijing 100083, People's Republic of China

2. School of Physics Science and Technology, Lingnan Normal University, Zhanjiang 524048, People's Republic of China 
Table S1. Optimized cartesian coordinate of porphyrin quarter-thiophenes fullerene system

\begin{tabular}{|c|c|c|c|}
\hline Charge & $\mathbf{0}$ & Multiplicity & 1 \\
\hline $\mathbf{C}$ & 9.79812300 & -1.53642300 & 3.16001500 \\
\hline $\mathbf{C}$ & 8.61872700 & -1.86572300 & 2.39409800 \\
\hline $\mathrm{C}$ & 8.74498500 & -2.25405400 & 1.04280500 \\
\hline C & 10.03850200 & -2.38117600 & 0.43878400 \\
\hline $\mathbf{C}$ & 11.16310200 & -2.04230900 & 1.16758600 \\
\hline $\mathrm{C}$ & 11.04832600 & -1.60697200 & 2.54240500 \\
\hline $\mathbf{C}$ & 9.47823200 & -0.41383300 & 4.02622800 \\
\hline $\mathbf{C}$ & 8.08446800 & -0.05938400 & 3.79412000 \\
\hline $\mathrm{C}$ & 7.55982600 & -0.96732100 & 2.78745000 \\
\hline $\mathbf{C}$ & 6.67677400 & -0.49350500 & 1.81402600 \\
\hline $\mathrm{C}$ & 7.79900300 & -1.80476500 & 0.06656100 \\
\hline $\mathbf{C}$ & 10.94585800 & -1.20629700 & -1.54467800 \\
\hline $\mathbf{C}$ & 12.13051200 & -0.85085400 & -0.76300600 \\
\hline C & 12.25580400 & -1.26638000 & 0.55098100 \\
\hline $\mathbf{C}$ & 12.78405300 & -0.35954000 & 1.55599000 \\
\hline $\mathbf{C}$ & 12.04339300 & -0.57334100 & 2.78775100 \\
\hline $\mathbf{C}$ & 11.73682700 & 0.50180000 & 3.62034000 \\
\hline $\mathbf{C}$ & 10.42474500 & 0.58691900 & 4.24757100 \\
\hline $\mathrm{C}$ & 7.69649100 & 1.28072700 & 3.79328900 \\
\hline $\mathbf{C}$ & 8.68079500 & 2.32241000 & 4.02659800 \\
\hline $\mathbf{C}$ & 10.02084200 & 1.98176800 & 4.24993200 \\
\hline $\mathrm{C}$ & 11.07920500 & 2.76063000 & 3.63314900 \\
\hline $\mathbf{C}$ & 12.14695800 & 1.84742500 & 3.24631100 \\
\hline $\mathrm{C}$ & 12.85173600 & 2.05697100 & 2.06338300 \\
\hline $\mathbf{C}$ & 13.18608300 & 0.93144600 & 1.20146700 \\
\hline $\mathbf{C}$ & 13.06029700 & 1.36678500 & -0.17785300 \\
\hline $\mathbf{C}$ & 12.54385500 & 0.49005000 & -1.13683000 \\
\hline $\mathbf{C}$ & 9.78550600 & 2.64657600 & -2.34571500 \\
\hline C & 8.84097300 & 1.66026600 & -2.57069700 \\
\hline $\mathrm{C}$ & 7.53075700 & 1.76447600 & -1.96987200 \\
\hline $\mathbf{C}$ & 7.21720900 & 2.85339400 & -1.14999500 \\
\hline C & 8.20891500 & 3.88494500 & -0.91225400 \\
\hline $\mathrm{C}$ & 10.66566200 & 4.12572300 & -0.74719500 \\
\hline $\mathbf{C}$ & 11.73003000 & 3.20983500 & -1.12996700 \\
\hline $\mathrm{C}$ & 11.19507900 & 2.28807400 & -2.11112200 \\
\hline $\mathbf{C}$ & 11.58978300 & 0.96152500 & -2.11437400 \\
\hline $\mathbf{C}$ & 10.60471400 & -0.11880600 & -2.33093700 \\
\hline C & 9.22257100 & 0.23228000 & -2.55985600 \\
\hline $\mathbf{C}$ & 7.13534900 & 0.41994500 & -1.59304100 \\
\hline $\mathrm{C}$ & 6.43705500 & 0.21492400 & -0.41685800 \\
\hline
\end{tabular}




\begin{tabular}{|c|c|c|c|}
\hline C & 6.11373800 & 1.33809800 & 0.44598000 \\
\hline $\mathrm{C}$ & 6.48935200 & 2.63595400 & 0.08700100 \\
\hline C & 7.02955500 & 3.53843300 & 1.09451900 \\
\hline C & 8.09455600 & 4.31514500 & 0.47319000 \\
\hline C & 9.23957600 & 4.63465200 & 1.20068600 \\
\hline $\mathrm{C}$ & 10.55207800 & 4.54502600 & 0.57718700 \\
\hline $\mathrm{C}$ & 12.64503400 & 2.75657400 & -0.17400600 \\
\hline $\mathrm{C}$ & 12.51850400 & 3.18969900 & 1.20926200 \\
\hline C & 11.49422600 & 4.06089800 & 1.57575500 \\
\hline C & 10.76045300 & 3.84563200 & 2.81498500 \\
\hline $\mathrm{C}$ & 9.37145000 & 4.19901600 & 2.58382300 \\
\hline C & 8.35200100 & 3.45421900 & 3.17904800 \\
\hline $\mathrm{C}$ & 7.15727400 & 3.11698900 & 2.41606400 \\
\hline C & 6.75700900 & 1.76883600 & 2.79182100 \\
\hline C & 6.25478500 & 0.90013100 & 1.82473900 \\
\hline C & 8.19297100 & -0.50724800 & -2.00168300 \\
\hline C & 6.79905900 & -0.92799500 & 0.44204400 \\
\hline $\mathrm{C}$ & 9.47135100 & 3.78453800 & -1.50600900 \\
\hline $\mathrm{C}$ & 9.95649800 & -2.24487000 & -1.08650600 \\
\hline C & 8.39478700 & -1.84320000 & -1.34319700 \\
\hline $\mathrm{C}$ & 10.08357900 & -3.59400300 & -1.83181300 \\
\hline $\mathbf{H}$ & 10.44839200 & -3.40563800 & -2.85999500 \\
\hline C & 7.86675900 & -3.04844800 & -2.20728600 \\
\hline $\mathbf{H}$ & 8.01549400 & -2.76899800 & -3.26889100 \\
\hline $\mathbf{N}$ & 8.74078900 & -4.15347000 & -1.82697500 \\
\hline H & 10.79051700 & -4.26757400 & -1.33810900 \\
\hline $\mathrm{C}$ & 8.61152400 & -5.32572700 & -2.67929600 \\
\hline H & 9.28068100 & -6.11224700 & -2.31692100 \\
\hline H & 7.58451900 & -5.69595200 & -2.63929300 \\
\hline $\mathbf{H}$ & 8.86577900 & -5.11065200 & -3.73427900 \\
\hline C & 6.42327700 & -3.34306100 & -1.95960400 \\
\hline $\mathrm{C}$ & 5.88061400 & -4.12213400 & -0.97272100 \\
\hline $\mathbf{S}$ & 5.18265400 & -2.51543800 & -2.85105800 \\
\hline C & 4.45568000 & -4.08945500 & -0.91554100 \\
\hline $\mathbf{H}$ & 6.49478200 & -4.70134700 & -0.29287100 \\
\hline C & 3.91341300 & -3.25632700 & -1.88331200 \\
\hline $\mathrm{C}$ & 2.53939300 & -2.93390300 & -2.19066900 \\
\hline C & 2.03806600 & -2.40004400 & -3.36349600 \\
\hline $\mathbf{S}$ & 1.24391300 & -3.11320400 & -1.01355300 \\
\hline $\mathrm{C}$ & 0.64828800 & -2.14527600 & -3.33129200 \\
\hline $\mathbf{H}$ & 2.66088900 & -2.21236200 & -4.23105000 \\
\hline C & 0.04987600 & -2.47887100 & -2.13160800 \\
\hline $\mathbf{H}$ & 0.09408300 & -1.74361100 & -4.17252200 \\
\hline
\end{tabular}




\begin{tabular}{|c|c|c|c|}
\hline C & -1.33336200 & -2.37323000 & -1.74603600 \\
\hline $\mathbf{C}$ & -1.94951600 & -2.80063500 & -0.58565400 \\
\hline $\mathbf{S}$ & -2.50553900 & -1.62450000 & -2.81529700 \\
\hline C & -3.33823400 & -2.54322000 & -0.55128400 \\
\hline $\mathbf{H}$ & -1.41449800 & -3.30312800 & 0.21276900 \\
\hline C & -3.82206900 & -1.91142600 & -1.68296500 \\
\hline $\mathbf{H}$ & -3.97902600 & -2.82618100 & 0.27630800 \\
\hline $\mathrm{C}$ & -5.17452800 & -1.47483600 & -1.93770800 \\
\hline C & -5.81254900 & -1.14819700 & -3.12695900 \\
\hline $\mathbf{S}$ & -6.27300700 & -1.31335100 & -0.57551600 \\
\hline $\mathrm{C}$ & -7.17627100 & -0.79156500 & -2.92225400 \\
\hline C & -7.59955400 & -0.84111000 & -1.61636000 \\
\hline $\mathbf{H}$ & -7.84344900 & -0.51902800 & -3.73279100 \\
\hline C & 3.67213500 & -4.88418800 & 0.09321000 \\
\hline H & 3.27772000 & -4.24770400 & 0.89543500 \\
\hline $\mathbf{H}$ & 2.81849300 & -5.39661000 & -0.36290300 \\
\hline $\mathbf{H}$ & 4.31145200 & -5.63970200 & 0.55960500 \\
\hline $\mathrm{C}$ & -5.18510200 & -1.17891100 & -4.49403500 \\
\hline $\mathbf{H}$ & -4.48942400 & -0.34352800 & -4.64345200 \\
\hline $\mathbf{H}$ & -4.62192600 & -2.10280400 & -4.66436400 \\
\hline $\mathbf{H}$ & -5.95591400 & -1.10561700 & -5.26697900 \\
\hline $\mathbf{C}$ & -8.92628200 & -0.49290200 & -1.07735100 \\
\hline C & -9.41070900 & 0.81070200 & -1.32745200 \\
\hline $\mathbf{C}$ & -9.61652200 & -1.48799300 & -0.36315700 \\
\hline $\mathbf{N}$ & -10.65670200 & 1.27635900 & -1.01610600 \\
\hline C & -8.60565200 & 1.85307800 & -1.95972900 \\
\hline $\mathbf{N}$ & -10.82036900 & -1.33960400 & 0.28421200 \\
\hline C & -9.22068800 & -2.85415500 & -0.18345400 \\
\hline C & -10.68262400 & 2.57240100 & -1.45775200 \\
\hline $\mathbf{C}$ & -9.39930300 & 2.94783300 & -2.04501400 \\
\hline $\mathbf{H}$ & -7.57344200 & 1.75476200 & -2.26019300 \\
\hline $\mathrm{C}$ & -11.20540500 & -2.52503400 & 0.87022600 \\
\hline C & -10.18176300 & -3.48121300 & 0.56540000 \\
\hline $\mathbf{H}$ & -8.32732000 & -3.29220300 & -0.60001300 \\
\hline $\mathrm{C}$ & -11.76159400 & 3.46944400 & -1.34782200 \\
\hline $\mathbf{H}$ & -9.14704100 & 3.92315000 & -2.43423500 \\
\hline C & -12.38380800 & -2.76204300 & 1.59151900 \\
\hline $\mathbf{H}$ & -10.20560600 & -4.51650700 & 0.86887800 \\
\hline C & -12.96910300 & 3.20904400 & -0.68348700 \\
\hline C & -13.42575800 & -1.83301300 & 1.77972000 \\
\hline $\mathbf{N}$ & -13.30425800 & 2.05442800 & -0.01488000 \\
\hline C & -14.08349800 & 4.10102800 & -0.54218300 \\
\hline $\mathbf{N}$ & -13.48032100 & -0.56807600 & 1.26306200 \\
\hline
\end{tabular}




\begin{tabular}{|c|c|c|c|}
\hline C & -14.62041800 & -2.12634300 & 2.56782600 \\
\hline $\mathrm{C}$ & -14.54922800 & 2.17095100 & 0.55986600 \\
\hline $\mathbf{C}$ & -15.03425500 & 3.47775200 & 0.22302000 \\
\hline $\mathbf{H}$ & -14.13006000 & 5.09053700 & -0.97011400 \\
\hline $\mathrm{C}$ & -14.67946400 & -0.05601200 & 1.67743800 \\
\hline $\mathbf{C}$ & -15.40656000 & -1.02516000 & 2.49297700 \\
\hline H & -14.81571100 & -3.04729300 & 3.09687100 \\
\hline $\mathrm{C}$ & -15.19640100 & 1.21225400 & 1.35140000 \\
\hline $\mathbf{H}$ & -15.98789800 & 3.87313600 & 0.53749900 \\
\hline H & -16.37971200 & -0.87158100 & 2.93538100 \\
\hline H & -11.37568600 & -0.48985000 & 0.28084100 \\
\hline $\mathbf{H}$ & -12.71886900 & 1.22688000 & 0.04228200 \\
\hline $\mathrm{C}$ & -12.53432800 & -4.12481400 & 2.18300800 \\
\hline C & -13.54987500 & -4.98696900 & 1.74469900 \\
\hline C & -11.65726200 & -4.57045700 & 3.18227100 \\
\hline $\mathrm{C}$ & -13.68684000 & -6.26097900 & 2.29404100 \\
\hline $\mathbf{H}$ & -14.22707500 & -4.65079700 & 0.96545900 \\
\hline C & -11.79370900 & -5.84466300 & 3.73204700 \\
\hline $\mathbf{H}$ & -10.87237700 & -3.90570200 & 3.53050300 \\
\hline C & -12.80919900 & -6.69365100 & 3.28957100 \\
\hline $\mathbf{H}$ & -14.47652800 & -6.91788300 & 1.94020400 \\
\hline $\mathbf{H}$ & -11.10943500 & -6.17183200 & 4.50991900 \\
\hline H & -12.91599900 & -7.68634300 & 3.71762200 \\
\hline $\mathrm{C}$ & -11.61804200 & 4.81888500 & -1.97123100 \\
\hline $\mathbf{C}$ & -11.49853800 & 4.94935900 & -3.36225900 \\
\hline C & -11.59533900 & 5.97754200 & -1.18171400 \\
\hline $\mathbf{C}$ & -11.36160300 & 6.20650400 & -3.94936500 \\
\hline H & -11.51787000 & 4.05580600 & -3.97880800 \\
\hline $\mathrm{C}$ & -11.45874000 & 7.23521100 & -1.76826400 \\
\hline $\mathbf{H}$ & -11.67515500 & 5.88322900 & -0.10287100 \\
\hline $\mathbf{C}$ & -11.34207700 & 7.35340100 & -3.15406200 \\
\hline $\mathbf{H}$ & -11.27407100 & 6.29014300 & -5.02904300 \\
\hline H & -11.43667700 & 8.12233000 & -1.14145900 \\
\hline H & -11.23501800 & 8.33299800 & -3.61121500 \\
\hline $\mathrm{C}$ & -16.53981900 & 1.58825000 & 1.88624300 \\
\hline C & -17.63279100 & 1.75136900 & 1.02375400 \\
\hline $\mathrm{C}$ & -16.72935100 & 1.78943900 & 3.26065600 \\
\hline C & -18.88552600 & 2.10671800 & 1.52315200 \\
\hline H & -17.49388800 & 1.58978600 & -0.04113100 \\
\hline $\mathrm{C}$ & -17.98200300 & 2.14281800 & 3.76083300 \\
\hline $\mathbf{H}$ & -15.88484500 & 1.66977400 & 3.93266700 \\
\hline C & -19.06372300 & 2.30313100 & 2.89333700 \\
\hline $\mathbf{H}$ & -19.72351300 & 2.22444300 & 0.84175100 \\
\hline
\end{tabular}




\begin{tabular}{llll}
\hline $\mathbf{H}$ & -18.11153800 & 2.29778800 & 4.82833500 \\
\hline $\mathbf{H}$ & -20.03953800 & 2.57903700 & 3.28285500 \\
\hline
\end{tabular}

Table S2. Optimized cartesian coordinate of porphyrin-octant -thiophenes fullerene system

\begin{tabular}{|c|c|c|c|}
\hline Charge & 0 & Multiplicity & 1 \\
\hline C & 17.13990400 & 0.27526900 & 3.55120600 \\
\hline $\mathbf{C}$ & 15.94185800 & -0.32345500 & 3.01599900 \\
\hline $\mathbf{C}$ & 16.00020300 & -1.06466600 & 1.81546000 \\
\hline $\mathbf{C}$ & 17.25203600 & -1.27410500 & 1.15061300 \\
\hline C & 18.39418000 & -0.67588200 & 1.65334500 \\
\hline $\mathbf{C}$ & 18.34383200 & 0.11338600 & 2.86226200 \\
\hline $\mathbf{C}$ & 16.79125300 & 1.56978900 & 4.10801500 \\
\hline $\mathbf{C}$ & 15.36147600 & 1.76055500 & 3.91981700 \\
\hline $\mathrm{C}$ & 14.84472700 & 0.58149000 & 3.24876600 \\
\hline $\mathbf{C}$ & 13.85727300 & 0.71285400 & 2.26933000 \\
\hline $\mathbf{C}$ & 14.94869300 & -0.96966700 & 0.84565400 \\
\hline $\mathbf{C}$ & 17.91182700 & -0.63729300 & -1.15037000 \\
\hline $\mathbf{C}$ & 19.11423300 & -0.00679700 & -0.60764300 \\
\hline $\mathbf{C}$ & 19.36912300 & -0.03511000 & 0.75280100 \\
\hline $\mathbf{C}$ & 19.89075400 & 1.14277600 & 1.42137400 \\
\hline $\mathbf{C}$ & 19.26296500 & 1.23209600 & 2.72689700 \\
\hline C & 18.92854300 & 2.47493300 & 3.26392300 \\
\hline $\mathbf{C}$ & 17.66422600 & 2.64918300 & 3.96308100 \\
\hline $\mathbf{C}$ & 14.86286900 & 3.02267500 & 3.59371000 \\
\hline $\mathbf{C}$ & 15.76889600 & 4.14547300 & 3.44570700 \\
\hline $\mathbf{C}$ & 17.14625900 & 3.96175100 & 3.62703800 \\
\hline $\mathbf{C}$ & 18.08667500 & 4.60266000 & 2.72845700 \\
\hline $\mathbf{C}$ & 19.19540800 & 3.68627400 & 2.50513300 \\
\hline C & 19.79255700 & 3.60400300 & 1.24873800 \\
\hline $\mathbf{C}$ & 20.15622600 & 2.30844600 & 0.69576200 \\
\hline $\mathbf{C}$ & 19.89457200 & 2.33758500 & -0.73012600 \\
\hline $\mathbf{C}$ & 19.38477200 & 1.19988700 & -1.36450000 \\
\hline C & 16.37720100 & 2.76587100 & -2.84674900 \\
\hline C & 15.50494200 & 1.69925500 & -2.70707600 \\
\hline $\mathbf{C}$ & 14.23970100 & 1.88524200 & -2.04009700 \\
\hline C & 13.89598600 & 3.13740000 & -1.51922800 \\
\hline $\mathbf{C}$ & 14.81012200 & 4.25207600 & -1.66209200 \\
\hline $\mathbf{C}$ & 17.24281900 & 4.67810400 & -1.79838900 \\
\hline $\mathbf{C}$ & 18.34895900 & 3.76009200 & -2.01673600 \\
\hline C & 17.82379200 & 2.57348100 & -2.65699000 \\
\hline $\mathrm{C}$ & 18.32699400 & 1.32370100 & -2.33777400 \\
\hline
\end{tabular}




\begin{tabular}{|c|c|c|c|}
\hline $\mathbf{C}$ & 17.42468700 & 0.16892900 & -2.16410600 \\
\hline $\mathrm{C}$ & 16.00359700 & 0.35719000 & -2.34909200 \\
\hline C & 13.98758300 & 0.67600900 & -1.28164500 \\
\hline C & 13.39635000 & 0.76163000 & -0.03347600 \\
\hline $\mathrm{C}$ & 13.04447600 & 2.05629800 & 0.51978600 \\
\hline $\mathbf{C}$ & 13.28140300 & 3.22591100 & -0.20914500 \\
\hline $\mathbf{C}$ & 13.81419700 & 4.40161500 & 0.46186700 \\
\hline $\mathrm{C}$ & 14.76087400 & 5.03960000 & -0.44128500 \\
\hline $\mathbf{C}$ & 15.92547100 & 5.61663200 & 0.06364700 \\
\hline $\mathbf{C}$ & 17.19069900 & 5.43902400 & -0.63096000 \\
\hline $\mathbf{C}$ & 19.36632600 & 3.64498900 & -1.06269900 \\
\hline $\mathbf{C}$ & 19.30449900 & 4.43357200 & 0.15667100 \\
\hline $\mathrm{C}$ & 18.23925100 & 5.30809200 & 0.36812400 \\
\hline $\mathbf{C}$ & 17.61880100 & 5.39859200 & 1.68066900 \\
\hline C & 16.19340900 & 5.58888100 & 1.49306600 \\
\hline $\mathrm{C}$ & 15.28575600 & 4.97627100 & 2.35974000 \\
\hline $\mathbf{C}$ & 14.07253400 & 4.37004100 & 1.83103500 \\
\hline $\mathrm{C}$ & 13.81664000 & 3.15698600 & 2.59071000 \\
\hline $\mathbf{C}$ & 13.32173000 & 2.02525700 & 1.94390000 \\
\hline $\mathbf{C}$ & 15.08232500 & -0.26478800 & -1.52277500 \\
\hline $\mathbf{C}$ & 13.91402500 & -0.07610000 & 1.06244700 \\
\hline $\mathbf{C}$ & 16.03065300 & 4.06876800 & -2.32160900 \\
\hline C & 17.05029900 & -1.57576300 & -0.34100500 \\
\hline $\mathrm{C}$ & 15.43791400 & -1.36533500 & -0.55395600 \\
\hline C & 17.25106900 & -3.06889600 & -0.70549000 \\
\hline $\mathbf{H}$ & 17.54494800 & -3.14571700 & -1.77077900 \\
\hline $\mathbf{C}$ & 14.96957300 & -2.79968300 & -1.03718100 \\
\hline $\mathbf{H}$ & 15.05185000 & -2.78965900 & -2.14283500 \\
\hline $\mathbf{N}$ & 15.96450900 & -3.70121800 & -0.45131800 \\
\hline $\mathbf{H}$ & 18.03970400 & -3.53382500 & -0.10470300 \\
\hline C & 15.90627000 & -5.06910300 & -0.95179600 \\
\hline $\mathbf{H}$ & 16.67155500 & -5.66906700 & -0.44865800 \\
\hline $\mathbf{H}$ & 14.92792100 & -5.50298400 & -0.73241900 \\
\hline $\mathbf{H}$ & 16.08044300 & -5.13053300 & -2.04294100 \\
\hline $\mathbf{C}$ & 13.56437000 & -3.17044300 & -0.66133200 \\
\hline $\mathbf{C}$ & 13.10298200 & -3.67777100 & 0.52506800 \\
\hline $\mathbf{S}$ & 12.24957400 & -2.95363400 & -1.78183000 \\
\hline $\mathbf{C}$ & 11.69668100 & -3.91495500 & 0.56576700 \\
\hline H & 13.76364000 & -3.89389300 & 1.35764100 \\
\hline $\mathrm{C}$ & 11.07445300 & -3.57499800 & -0.62780000 \\
\hline $\mathbf{C}$ & 9.68744500 & -3.66026700 & -1.03720400 \\
\hline C & 9.18378200 & -3.67267500 & -2.32518200 \\
\hline $\mathbf{S}$ & 8.35878100 & -3.69617900 & 0.11703700 \\
\hline
\end{tabular}




\begin{tabular}{|c|c|c|c|}
\hline C & 7.77319500 & -3.72504600 & -2.39427200 \\
\hline $\mathbf{H}$ & 9.82226900 & -3.66665600 & -3.20212400 \\
\hline C & 7.15502800 & -3.75580600 & -1.15900600 \\
\hline H & 7.22353100 & -3.76886200 & -3.32857500 \\
\hline $\mathrm{C}$ & 5.74939200 & -3.83335500 & -0.84212100 \\
\hline $\mathbf{C}$ & 5.14161700 & -4.11629000 & 0.36624500 \\
\hline $\mathbf{S}$ & 4.53292300 & -3.55812500 & -2.07733300 \\
\hline $\mathrm{C}$ & 3.73119400 & -4.12870400 & 0.30696100 \\
\hline $\mathbf{H}$ & 5.70168100 & -4.33730500 & 1.26873000 \\
\hline $\mathbf{C}$ & 3.21323500 & -3.85438600 & -0.94778400 \\
\hline $\mathbf{H}$ & 3.10305300 & -4.35699200 & 1.16124800 \\
\hline $\mathrm{C}$ & 1.82220500 & -3.76259200 & -1.32585900 \\
\hline $\mathrm{C}$ & 1.20701800 & -3.78599500 & -2.57311000 \\
\hline $\mathbf{S}$ & 0.62237300 & -3.59561200 & -0.04585000 \\
\hline C & -0.20710300 & -3.69787800 & -2.47741700 \\
\hline $\mathrm{C}$ & -0.70186100 & -3.60332400 & -1.19323500 \\
\hline $\mathbf{H}$ & -0.85122900 & -3.72980100 & -3.35072700 \\
\hline $\mathbf{C}$ & 11.01839300 & -4.48594000 & 1.78449600 \\
\hline $\mathbf{H}$ & 10.46673300 & -3.71950900 & 2.34417600 \\
\hline $\mathbf{H}$ & 10.30415800 & -5.27514500 & 1.52613900 \\
\hline $\mathbf{H}$ & 11.76076200 & -4.91380700 & 2.46551000 \\
\hline $\mathbf{C}$ & 1.91049400 & -3.91361400 & -3.89951100 \\
\hline $\mathbf{H}$ & 2.44661100 & -2.99481900 & -4.16939900 \\
\hline $\mathbf{H}$ & 2.64324000 & -4.72820000 & -3.89880000 \\
\hline $\mathbf{H}$ & 1.18659700 & -4.11481100 & -4.69512500 \\
\hline $\mathbf{C}$ & -2.07007400 & -3.51116600 & -0.74945100 \\
\hline $\mathbf{C}$ & -2.58172900 & -3.64813300 & 0.52420400 \\
\hline $\mathbf{S}$ & -3.36770500 & -3.17244900 & -1.87721700 \\
\hline $\mathrm{C}$ & -3.99132200 & -3.51029800 & 0.62536300 \\
\hline H & -1.95726400 & -3.87179700 & 1.38364500 \\
\hline $\mathbf{C}$ & -4.58643900 & -3.26334400 & -0.60736400 \\
\hline $\mathbf{C}$ & -5.97154400 & -3.08593700 & -0.97683300 \\
\hline $\mathrm{C}$ & -6.52462700 & -3.15473700 & -2.24449500 \\
\hline $\mathbf{S}$ & -7.22555400 & -2.67492100 & 0.19086100 \\
\hline $\mathbf{C}$ & -7.91280000 & -2.89998900 & -2.28495800 \\
\hline $\mathbf{H}$ & -5.94139300 & -3.40732100 & -3.12347600 \\
\hline $\mathbf{C}$ & -8.46767100 & -2.62900800 & -1.04858800 \\
\hline H & -8.49948600 & -2.94089500 & -3.19661300 \\
\hline $\mathbf{C}$ & -9.84034800 & -2.34556200 & -0.70614800 \\
\hline $\mathrm{C}$ & -10.44023100 & -2.30836100 & 0.53794600 \\
\hline $\mathbf{S}$ & -11.02321100 & -1.99114700 & -1.95413900 \\
\hline $\mathbf{C}$ & -11.82243100 & -2.01786200 & 0.49705000 \\
\hline $\mathbf{H}$ & -9.90174300 & -2.51342200 & 1.45720100 \\
\hline
\end{tabular}




\begin{tabular}{|c|c|c|c|}
\hline C & -12.32251800 & -1.82348200 & -0.77789400 \\
\hline $\mathbf{H}$ & -12.44763700 & -1.97224200 & 1.38222900 \\
\hline $\mathbf{C}$ & -13.67737400 & -1.47480700 & -1.15386400 \\
\hline C & -14.35681200 & -1.62072400 & -2.35558900 \\
\hline $\mathbf{S}$ & -14.71905900 & -0.74487400 & 0.06181100 \\
\hline C & -15.70389900 & -1.15911000 & -2.26868500 \\
\hline $\mathbf{C}$ & -16.07718800 & -0.67205300 & -1.04131600 \\
\hline $\mathbf{H}$ & -16.39493200 & -1.20612500 & -3.10441500 \\
\hline $\mathbf{C}$ & -4.71388400 & -3.65008500 & 1.94028000 \\
\hline $\mathbf{H}$ & -5.08778800 & -2.68580500 & 2.30739300 \\
\hline $\mathbf{H}$ & -5.57419600 & -4.32440400 & 1.86621600 \\
\hline $\mathbf{H}$ & -4.03902400 & -4.05023600 & 2.70316900 \\
\hline $\mathbf{C}$ & -13.79568400 & -2.21408500 & -3.62237100 \\
\hline $\mathbf{H}$ & -13.12873300 & -1.51505900 & -4.14367700 \\
\hline $\mathbf{H}$ & -13.22083000 & -3.12623600 & -3.42929000 \\
\hline $\mathbf{H}$ & -14.60536600 & -2.46740100 & -4.31395200 \\
\hline $\mathbf{C}$ & -17.38470000 & -0.10063800 & -0.63936300 \\
\hline $\mathbf{C}$ & -18.13720800 & -0.82301000 & 0.30567800 \\
\hline $\mathbf{C}$ & -17.77794000 & 1.12121200 & -1.23535600 \\
\hline $\mathbf{N}$ & -19.35916600 & -0.46401900 & 0.82288400 \\
\hline $\mathbf{C}$ & -17.79012800 & -2.07549200 & 0.91188700 \\
\hline $\mathbf{N}$ & -18.96476400 & 1.76497200 & -1.03588600 \\
\hline $\mathbf{C}$ & -16.91679800 & 1.87689100 & -2.14076900 \\
\hline $\mathbf{C}$ & -19.80389000 & -1.40751900 & 1.72277300 \\
\hline $\mathrm{C}$ & -18.79193700 & -2.42251800 & 1.77772400 \\
\hline $\mathbf{H}$ & -16.88789500 & -2.62929900 & 0.70175700 \\
\hline $\mathbf{C}$ & -18.89327400 & 2.90168700 & -1.79471900 \\
\hline $\mathbf{C}$ & -17.61226300 & 2.98365200 & -2.49092200 \\
\hline $\mathbf{H}$ & -15.91625600 & 1.59916800 & -2.43813800 \\
\hline $\mathbf{C}$ & -21.00800600 & -1.37945800 & 2.44357800 \\
\hline $\mathbf{H}$ & -18.84098500 & -3.30157000 & 2.40239700 \\
\hline $\mathbf{C}$ & -19.87822900 & 3.90650500 & -1.88678600 \\
\hline $\mathbf{H}$ & -17.29316500 & 3.79187600 & -3.13291800 \\
\hline $\mathbf{C}$ & -21.98737300 & -0.36475400 & 2.36396300 \\
\hline $\mathbf{C}$ & -21.08599400 & 3.93240500 & -1.16707800 \\
\hline $\mathbf{N}$ & -21.91318200 & 0.76894800 & 1.60548100 \\
\hline $\mathbf{C}$ & -23.25905800 & -0.43417100 & 3.07754100 \\
\hline $\mathbf{N}$ & -21.51820900 & 2.99936800 & -0.25345000 \\
\hline $\mathbf{C}$ & -22.10826400 & 4.93547600 & -1.23769200 \\
\hline $\mathbf{C}$ & -23.09253600 & 1.42691300 & 1.81924800 \\
\hline $\mathbf{C}$ & -23.94696400 & 0.68156300 & 2.73867200 \\
\hline $\mathbf{H}$ & -23.57673500 & -1.23813000 & 3.72562800 \\
\hline $\mathbf{C}$ & -22.74411400 & 3.35372300 & 0.26243900 \\
\hline
\end{tabular}




\begin{tabular}{|c|c|c|c|}
\hline $\mathbf{C}$ & -23.10544700 & 4.59175300 & -0.36401500 \\
\hline $\mathbf{H}$ & -22.06935400 & 5.80425400 & -1.87728000 \\
\hline $\mathbf{C}$ & -23.48780900 & 2.64142500 & 1.21854100 \\
\hline H & -24.93910900 & 0.97048500 & 3.05403300 \\
\hline $\mathbf{H}$ & -24.01531000 & 5.13670300 & -0.16242900 \\
\hline $\mathbf{H}$ & -19.86615400 & 0.37823000 & 0.56995100 \\
\hline $\mathbf{H}$ & -21.00463600 & 2.16340100 & 0.00687100 \\
\hline $\mathbf{C}$ & -24.80734600 & 3.23118200 & 1.61577500 \\
\hline $\mathbf{C}$ & -25.87735100 & 3.27995600 & 0.70854400 \\
\hline $\mathbf{C}$ & -24.99926600 & 3.74801000 & 2.90670100 \\
\hline $\mathbf{C}$ & -27.10473400 & 3.82951600 & 1.08152500 \\
\hline $\mathbf{H}$ & -25.74445000 & 2.87433500 & -0.29061500 \\
\hline $\mathbf{C}$ & -26.22596600 & 4.29848100 & 3.27970100 \\
\hline $\mathbf{H}$ & -24.17681400 & 3.72000300 & 3.61595200 \\
\hline $\mathrm{C}$ & -27.28264400 & 4.34108000 & 2.36819100 \\
\hline $\mathbf{H}$ & -27.92341400 & 3.85282100 & 0.36701900 \\
\hline $\mathbf{H}$ & -26.35418900 & 4.69736700 & 4.28256000 \\
\hline $\mathbf{H}$ & -28.23827700 & 4.76913600 & 2.65883600 \\
\hline $\mathbf{C}$ & -21.26257000 & -2.53479600 & 3.36514500 \\
\hline $\mathbf{C}$ & -21.53245700 & -3.81517100 & 2.85758300 \\
\hline $\mathbf{C}$ & -21.23607500 & -2.35719400 & 4.75715800 \\
\hline $\mathbf{C}$ & -21.76943400 & -4.88832800 & 3.71782800 \\
\hline $\mathbf{H}$ & -21.56478700 & -3.96327000 & 1.78166700 \\
\hline $\mathbf{C}$ & -21.47226000 & -3.42998100 & 5.61764300 \\
\hline $\mathbf{H}$ & -21.02394000 & -1.37134900 & 5.16141700 \\
\hline $\mathbf{C}$ & -21.73952500 & -4.69903600 & 5.10060800 \\
\hline $\mathbf{H}$ & -21.98225300 & -5.87143400 & 3.30611200 \\
\hline $\mathbf{H}$ & -21.44378600 & -3.27389100 & 6.69286400 \\
\hline $\mathbf{H}$ & -21.92399200 & -5.53445600 & 5.77067500 \\
\hline $\mathbf{C}$ & -19.63073000 & 5.05544400 & -2.81786200 \\
\hline $\mathbf{C}$ & -19.63687500 & 4.86198900 & -4.20806600 \\
\hline $\mathbf{C}$ & -19.38765900 & 6.34583600 & -2.32187300 \\
\hline $\mathbf{C}$ & -19.40703100 & 5.92862100 & -5.07787600 \\
\hline $\mathbf{H}$ & -19.82803800 & 3.86831000 & -4.60350400 \\
\hline $\mathbf{C}$ & -19.15680300 & 7.41277000 & -3.19135300 \\
\hline $\mathbf{H}$ & -19.37056800 & 6.50662200 & -1.24744800 \\
\hline $\mathbf{C}$ & -19.16641800 & 7.20750600 & -4.57219200 \\
\hline $\mathbf{H}$ & -19.41931600 & 5.75994100 & -6.15151100 \\
\hline $\mathbf{H}$ & -18.96415700 & 8.40359200 & -2.78831700 \\
\hline $\mathbf{H}$ & -18.98656500 & 8.03813900 & -5.24942200 \\
\hline
\end{tabular}


Table S3 Optimization Information of porphyrin quarter-thiophenes fullerene compound

\begin{tabular}{ccccc}
\hline & SCF Energy & \multicolumn{2}{c}{$\mathbf{- 6 4 2 7 . 4 6 3 9 0 6}$} & Hartree \\
\hline & & & & \\
\hline Item & Value & Threshold & Converged? \\
\hline RMS & Force & 0.000005 & 0.000450 & YES \\
\hline Maximum & Displacement & 0.000001 & 0.000300 & YES \\
\hline RMS & Displacement & 0.001346 & 0.001800 & YES \\
\hline & & & 0.001200 & YES \\
\hline
\end{tabular}

Table S4 Optimization Information of porphyrin octant -thiophenes fullerene compound

\begin{tabular}{ccccc}
\hline \multicolumn{2}{c}{ SCF Energy } & \multicolumn{2}{c}{$\mathbf{8 7 1 2 . 7 0 5 7 4 8}$} & Hartree \\
\hline & & & & \\
\hline Item & Value & Threshold & Converged? \\
\hline Maximum & Force & 0.000003 & 0.000450 & YES \\
\hline RMS & Force & 0.000000 & 0.000300 & YES \\
\hline Maximum & Displacement & 0.000069 & 0.001800 & YES \\
\hline RMS & Displacement & 0.000249 & 0.001200 & YES \\
\hline
\end{tabular}

Table S5 Vibration and thermodynamics analysis of porphyrin quarter-thiophenes fullerene compound

\begin{tabular}{|lr|}
\hline Zero-point correction (Hartree/Particle) & 1.244335 \\
\hline Thermal correction to Energy (Hartree/Particle) & 1.323421 \\
\hline Thermal correction to Enthalpy (Hartree/Particle) & 1.324365 \\
\hline Thermal correction to Gibbs Free Energy (Hartree/Particle) & 1.121281 \\
\hline Sum of electronic and zero-point Energies (Hartree/Particle) & -6426.219572 \\
\hline Sum of electronic and thermal Energies (Hartree/Particle) & -6426.140486 \\
\hline Sum of electronic and thermal Enthalpies (Hartree/Particle) & -6426.139542 \\
\hline Sum of electronic and thermal Free Energies (Hartree/Particle) & -6426.342626 \\
\hline
\end{tabular}

Table S6 Vibration and thermodynamics analysis of porphyrin octant -thiophenes fullerene compound

\begin{tabular}{lc}
\hline Zero-point correction (Hartree/Particle) & 1.484667 \\
\hline Thermal correction to Energy (Hartree/Particle) & 1.586129 \\
\hline Thermal correction to Enthalpy (Hartree/Particle) & 1.587073 \\
\hline Thermal correction to Gibbs Free Energy (Hartree/Particle) & 1.328374 \\
\hline Sum of electronic and zero-point Energies (Hartree/Particle) & -8711.221082 \\
\hline Sum of electronic and thermal Energies (Hartree/Particle) & -8711.119619 \\
\hline Sum of electronic and thermal Enthalpies (Hartree/Particle) & -8711.118675 \\
\hline Sum of electronic and thermal Free Energies (Hartree/Particle) & -8711.377374 \\
\hline
\end{tabular}



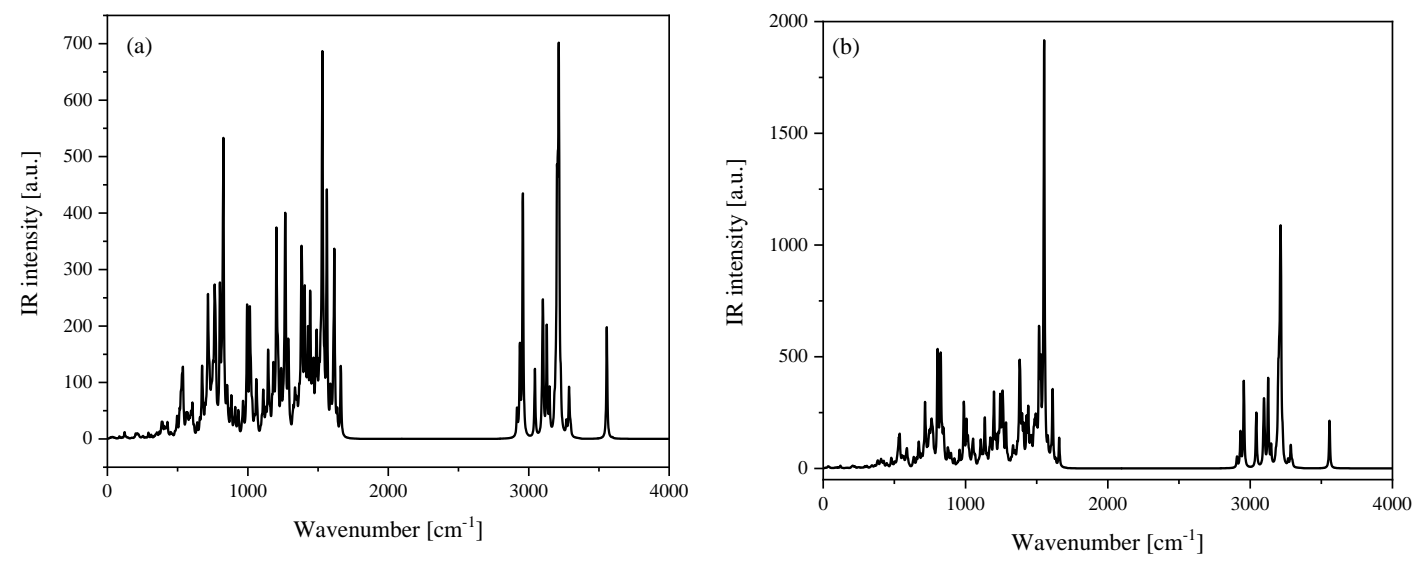

Figure S1 The infrared spectroscopy of (a) porphyrin quarter-thiophenes-fullerene, and (b) porphyrin octant--thiophenes-fullerene.

Table S7. Excited states information of porphyrin quarter-thiophenes fullerene compound

\begin{tabular}{|c|c|c|c|c|c|c|c|}
\hline $\begin{array}{l}\text { Excited } \\
\text { States }\end{array}$ & $\begin{array}{c}\text { Excitation } \\
\text { Energy } \\
{[\mathrm{eV}]}\end{array}$ & $\begin{array}{c}\text { Absorption } \\
\text { Wavelength } \\
{[\mathrm{nm}]}\end{array}$ & $\begin{array}{l}\text { Oscillator } \\
\text { Strength }\end{array}$ & $\begin{array}{l}\text { Excited } \\
\text { States }\end{array}$ & $\begin{array}{c}\text { Excitation } \\
\text { Energy } \\
{[\mathrm{eV}]}\end{array}$ & $\begin{array}{c}\text { Absorption } \\
\text { Wavelength } \\
{[\mathrm{nm}]}\end{array}$ & $\begin{array}{c}\text { Oscillator } \\
\text { Strength }\end{array}$ \\
\hline 1 & 1.09 & 1137.1 & $\mathrm{f}=0.0250$ & 100 & 3.4344 & 361.01 & $f=0.0007$ \\
\hline 2 & 1.251 & 991.44 & $f=0.0063$ & 101 & 3.4495 & 359.43 & $f=0.0038$ \\
\hline 3 & 1.455 & 851.86 & $\mathrm{f}=0.0040$ & 102 & 3.4668 & 357.63 & $f=0.0004$ \\
\hline 4 & 1.474 & 841.25 & $\mathrm{f}=0.0017$ & 103 & 3.4696 & 357.34 & $\mathrm{f}=0.0006$ \\
\hline 5 & 1.643 & 754.83 & $\mathrm{f}=0.0013$ & 104 & 3.4811 & 356.16 & $\mathrm{f}=0.0003$ \\
\hline 6 & 1.696 & 730.98 & $\mathrm{f}=0.0054$ & 105 & 3.4823 & 356.04 & $\mathrm{f}=0.0043$ \\
\hline 7 & 1.716 & 722.52 & $\mathrm{f}=0.0000$ & 106 & 3.4908 & 355.18 & $f=0.0014$ \\
\hline 8 & 1.749 & 708.93 & $\mathrm{f}=0.0017$ & 107 & 3.5124 & 352.99 & $\mathrm{f}=0.0030$ \\
\hline 9 & 1.78 & 696.4 & $\mathrm{f}=0.0011$ & 108 & 3.5148 & 352.75 & $\mathrm{f}=0.0035$ \\
\hline 10 & 1.81 & 684.96 & $\mathrm{f}=0.0010$ & 109 & 3.5302 & 351.21 & $\mathrm{f}=0.2537$ \\
\hline 11 & 1.832 & 676.68 & $\mathrm{f}=0.0002$ & 110 & 3.5346 & 350.77 & $\mathrm{f}=0.0026$ \\
\hline 12 & 1.88 & 659.57 & $\mathrm{f}=0.0055$ & 111 & 3.5364 & 350.6 & $\mathrm{f}=0.0282$ \\
\hline 13 & 2.051 & 604.61 & $\mathrm{f}=0.2463$ & 112 & 3.5518 & 349.07 & $\mathrm{f}=0.0025$ \\
\hline 14 & 2.053 & 603.98 & $\mathrm{f}=0.1694$ & 113 & 3.5661 & 347.67 & $\mathrm{f}=0.0185$ \\
\hline 15 & 2.063 & 601.09 & $\mathrm{f}=0.0001$ & 114 & 3.5716 & 347.14 & $\mathrm{f}=0.0054$ \\
\hline 16 & 2.086 & 594.24 & $\mathrm{f}=0.0001$ & 115 & 3.5776 & 346.56 & $\mathrm{f}=0.3946$ \\
\hline 17 & 2.137 & 580.22 & $\mathrm{f}=0.2370$ & 116 & 3.5779 & 346.53 & $\mathrm{f}=0.1136$ \\
\hline 18 & 2.149 & 576.85 & $\mathrm{f}=0.0011$ & 117 & 3.5852 & 345.82 & $\mathrm{f}=0.0089$ \\
\hline 19 & 2.168 & 572.02 & $\mathrm{f}=0.0014$ & 118 & 3.5978 & 344.61 & $\mathrm{f}=0.0011$ \\
\hline 20 & 2.177 & 569.53 & $\mathrm{f}=0.0035$ & 119 & 3.6018 & 344.23 & $\mathrm{f}=0.0036$ \\
\hline 21 & 2.233 & 555.17 & $\mathrm{f}=0.0014$ & 120 & 3.6215 & 342.35 & $\mathrm{f}=0.0000$ \\
\hline 22 & 2.263 & 547.94 & $\mathrm{f}=0.0109$ & 121 & 3.6264 & 341.89 & $\mathrm{f}=0.0057$ \\
\hline 23 & 2.305 & 537.89 & $\mathrm{f}=0.0000$ & 122 & 3.6307 & 341.49 & $\mathrm{f}=0.0009$ \\
\hline 24 & 2.312 & 536.21 & $\mathrm{f}=0.0016$ & 123 & 3.6314 & 341.43 & $\mathrm{f}=0.0079$ \\
\hline
\end{tabular}




\begin{tabular}{|c|c|c|c|c|c|c|c|}
\hline 25 & 2.339 & 530.04 & $\mathrm{f}=0.0066$ & 124 & 3.6408 & 340.54 & $\mathrm{f}=0.0073$ \\
\hline 26 & 2.35 & 527.54 & $\mathrm{f}=0.1585$ & 125 & 3.6551 & 339.21 & $\mathrm{f}=0.0182$ \\
\hline 27 & 2.423 & 511.77 & $\mathrm{f}=0.0160$ & 126 & 3.6615 & 338.62 & $f=0.0042$ \\
\hline 28 & 2.444 & 507.28 & $\mathrm{f}=0.0035$ & 127 & 3.6717 & 337.67 & $\mathrm{f}=0.0142$ \\
\hline 29 & 2.449 & 506.22 & $\mathrm{f}=0.0150$ & 128 & 3.6776 & 337.13 & $\mathrm{f}=0.0124$ \\
\hline 30 & 2.546 & 486.99 & $\mathrm{f}=0.0083$ & 129 & 3.6839 & 336.56 & $f=0.0022$ \\
\hline 31 & 2.566 & 483.17 & $\mathrm{f}=0.0006$ & 130 & 3.692 & 335.82 & $f=0.0061$ \\
\hline 32 & 2.609 & 475.22 & $\mathrm{f}=0.1392$ & 131 & 3.6993 & 335.15 & $\mathrm{f}=0.0141$ \\
\hline 33 & 2.616 & 473.93 & $\mathrm{f}=0.1340$ & 132 & 3.7049 & 334.65 & $\mathrm{f}=0.0000$ \\
\hline 34 & 2.62 & 473.14 & $\mathrm{f}=0.8100$ & 133 & 3.7071 & 334.45 & $\mathrm{f}=0.0070$ \\
\hline 35 & 2.627 & 472.04 & $\mathrm{f}=0.2812$ & 134 & 3.7237 & 332.96 & $\mathrm{f}=0.0008$ \\
\hline 36 & 2.656 & 466.85 & $\mathrm{f}=0.0313$ & 135 & 3.7255 & 332.8 & $\mathrm{f}=0.0029$ \\
\hline 37 & 2.69 & 460.98 & $\mathrm{f}=0.0001$ & 136 & 3.7267 & 332.69 & $\mathrm{f}=0.0000$ \\
\hline 38 & 2.731 & 454.01 & $\mathrm{f}=0.0012$ & 137 & 3.7337 & 332.06 & $\mathrm{f}=0.0002$ \\
\hline 39 & 2.788 & 444.65 & $\mathrm{f}=0.0000$ & 138 & 3.7362 & 331.85 & $f=0.0001$ \\
\hline 40 & 2.802 & 442.55 & $\mathrm{f}=0.0001$ & 139 & 3.7424 & 331.29 & $f=0.0001$ \\
\hline 41 & 2.806 & 441.93 & $\mathrm{f}=0.0038$ & 140 & 3.7439 & 331.16 & $\mathrm{f}=0.0001$ \\
\hline 42 & 2.814 & 440.55 & $\mathrm{f}=0.0036$ & 141 & 3.764 & 329.4 & $\mathrm{f}=0.0002$ \\
\hline 43 & 2.849 & 435.13 & $\mathrm{f}=0.0041$ & 142 & 3.7671 & 329.13 & $\mathrm{f}=0.0008$ \\
\hline 44 & 2.859 & 433.61 & $\mathrm{f}=0.0009$ & 143 & 3.77 & 328.87 & $\mathrm{f}=0.0004$ \\
\hline 45 & 2.862 & 433.16 & $\mathrm{f}=0.0059$ & 144 & 3.7716 & 328.73 & $\mathrm{f}=0.0003$ \\
\hline 46 & 2.896 & 428.1 & $\mathrm{f}=0.0039$ & 145 & 3.7736 & 328.56 & $\mathrm{f}=0.0013$ \\
\hline 47 & 2.907 & 426.56 & $\mathrm{f}=0.0000$ & 146 & 3.7847 & 327.59 & $\mathrm{f}=0.0000$ \\
\hline 48 & 2.915 & 425.33 & $\mathrm{f}=0.0045$ & 147 & 3.789 & 327.22 & $\mathrm{f}=0.0006$ \\
\hline 49 & 2.945 & 420.98 & $\mathrm{f}=0.0119$ & 148 & 3.7986 & 326.4 & $\mathrm{f}=0.0005$ \\
\hline 50 & 2.971 & 417.38 & $\mathrm{f}=0.4351$ & 149 & 3.8068 & 325.7 & $\mathrm{f}=0.0015$ \\
\hline 51 & 2.99 & 414.61 & $\mathrm{f}=0.0033$ & 150 & 3.81 & 325.42 & $f=0.0005$ \\
\hline 52 & 3.011 & 411.77 & $\mathrm{f}=0.0149$ & 151 & 3.8227 & 324.33 & $\mathrm{f}=0.0021$ \\
\hline 53 & 3.016 & 411.14 & $\mathrm{f}=0.0059$ & 152 & 3.8257 & 324.08 & $\mathrm{f}=0.0000$ \\
\hline 54 & 3.018 & 410.77 & $\mathrm{f}=0.0002$ & 153 & 3.8273 & 323.94 & $\mathrm{f}=0.0003$ \\
\hline 55 & 3.055 & 405.86 & $\mathrm{f}=0.0016$ & 154 & 3.8334 & 323.43 & $\mathrm{f}=0.0000$ \\
\hline 56 & 3.06 & 405.18 & $\mathrm{f}=0.0000$ & 155 & 3.8421 & 322.7 & $\mathrm{f}=0.0003$ \\
\hline 57 & 3.065 & 404.47 & $\mathrm{f}=0.3846$ & 156 & 3.8437 & 322.57 & $\mathrm{f}=0.0157$ \\
\hline 58 & 3.066 & 404.35 & $\mathrm{f}=0.0339$ & 157 & 3.8678 & 320.55 & $\mathrm{f}=0.0449$ \\
\hline 59 & 3.086 & 401.81 & $\mathrm{f}=0.0002$ & 158 & 3.871 & 320.29 & $\mathrm{f}=0.0280$ \\
\hline 60 & 3.103 & 399.62 & $\mathrm{f}=0.0002$ & 159 & 3.8791 & 319.62 & $\mathrm{f}=0.0117$ \\
\hline 61 & 3.141 & 394.78 & $\mathrm{f}=0.0040$ & 160 & 3.885 & 319.13 & $\mathrm{f}=0.0071$ \\
\hline 62 & 3.144 & 394.34 & $\mathrm{f}=0.0017$ & 161 & 3.8874 & 318.94 & $\mathrm{f}=0.0172$ \\
\hline 63 & 3.159 & 392.5 & $\mathrm{f}=0.0078$ & 162 & 3.8917 & 318.58 & $\mathrm{f}=0.0421$ \\
\hline 64 & 3.161 & 392.3 & $\mathrm{f}=0.2574$ & 163 & 3.9001 & 317.9 & $\mathrm{f}=0.0036$ \\
\hline 65 & 3.165 & 391.69 & $\mathrm{f}=0.0003$ & 164 & 3.9028 & 317.68 & $\mathrm{f}=0.0025$ \\
\hline 66 & 3.172 & 390.86 & $\mathrm{f}=0.0032$ & 165 & 3.9061 & 317.41 & $\mathrm{f}=0.0001$ \\
\hline 67 & 3.177 & 390.3 & $\mathrm{f}=0.0035$ & 166 & 3.9209 & 316.21 & $\mathrm{f}=0.0298$ \\
\hline
\end{tabular}




\begin{tabular}{|c|c|c|c|c|c|c|c|}
\hline 68 & 3.191 & 388.52 & $\mathrm{f}=0.0091$ & 167 & 3.9233 & 316.02 & $\mathrm{f}=0.0012$ \\
\hline 69 & 3.199 & 387.55 & $\mathrm{f}=0.0041$ & 168 & 3.9234 & 316.02 & $\mathrm{f}=0.0103$ \\
\hline 70 & 3.204 & 386.93 & $\mathrm{f}=0.1261$ & 169 & 3.9322 & 315.31 & $\mathrm{f}=0.0005$ \\
\hline 71 & 3.209 & 386.36 & $\mathrm{f}=0.0010$ & 170 & 3.9354 & 315.05 & $\mathrm{f}=0.0047$ \\
\hline 72 & 3.216 & 385.54 & $\mathrm{f}=0.0016$ & 171 & 3.9404 & 314.65 & $\mathrm{f}=0.0005$ \\
\hline 73 & 3.222 & 384.75 & $\mathrm{f}=0.0078$ & 172 & 3.9446 & 314.31 & $\mathrm{f}=0.0000$ \\
\hline 74 & 3.228 & 384.15 & $\mathrm{f}=0.0019$ & 173 & 3.9478 & 314.06 & $\mathrm{f}=0.0000$ \\
\hline 75 & 3.249 & 381.58 & $\mathrm{f}=0.0016$ & 174 & 3.9599 & 313.1 & $\mathrm{f}=0.0198$ \\
\hline 76 & 3.263 & 379.98 & $\mathrm{f}=0.2776$ & 175 & 3.9617 & 312.95 & $\mathrm{f}=0.0015$ \\
\hline 77 & 3.269 & 379.33 & $\mathrm{f}=0.0395$ & 176 & 3.9699 & 312.31 & $\mathrm{f}=0.0069$ \\
\hline 78 & 3.273 & 378.82 & $\mathrm{f}=0.0019$ & 177 & 3.9747 & 311.93 & $\mathrm{f}=0.0047$ \\
\hline 79 & 3.274 & 378.74 & $\mathrm{f}=0.0598$ & 178 & 3.9821 & 311.36 & $\mathrm{f}=0.0027$ \\
\hline 80 & 3.279 & 378.09 & $\mathrm{f}=0.0026$ & 179 & 3.9828 & 311.3 & $\mathrm{f}=0.0012$ \\
\hline 81 & 3.288 & 377.08 & $\mathrm{f}=0.0061$ & 180 & 3.9908 & 310.67 & $\mathrm{f}=0.0031$ \\
\hline 82 & 3.297 & 376.1 & $\mathrm{f}=0.0031$ & 181 & 3.9921 & 310.57 & $f=0.0005$ \\
\hline 83 & 3.31 & 374.53 & $\mathrm{f}=0.0028$ & 182 & 3.9971 & 310.19 & $\mathrm{f}=0.0146$ \\
\hline 84 & 3.312 & 374.34 & $\mathrm{f}=0.0007$ & 183 & 4.0013 & 309.86 & $\mathrm{f}=0.0019$ \\
\hline 85 & 3.319 & 373.52 & $\mathrm{f}=0.0046$ & 184 & 4.0025 & 309.77 & $\mathrm{f}=0.0255$ \\
\hline 86 & 3.324 & 373.05 & $\mathrm{f}=0.2336$ & 185 & 4.0041 & 309.64 & $\mathrm{f}=0.0042$ \\
\hline 87 & 3.334 & 371.84 & $\mathrm{f}=0.0000$ & 186 & 4.0144 & 308.85 & $\mathrm{f}=0.0001$ \\
\hline 88 & 3.345 & 370.71 & $\mathrm{f}=0.0083$ & 187 & 4.0168 & 308.66 & $\mathrm{f}=0.0013$ \\
\hline 89 & 3.357 & 369.33 & $\mathrm{f}=0.0000$ & 188 & 4.0196 & 308.45 & $\mathrm{f}=0.0010$ \\
\hline 90 & 3.367 & 368.29 & $\mathrm{f}=0.0012$ & 189 & 4.0241 & 308.11 & $\mathrm{f}=0.0004$ \\
\hline 91 & 3.371 & 367.79 & $\mathrm{f}=0.0017$ & 190 & 4.0262 & 307.94 & $\mathrm{f}=0.0004$ \\
\hline 92 & 3.377 & 367.1 & $\mathrm{f}=0.0001$ & 191 & 4.0397 & 306.92 & $\mathrm{f}=0.0012$ \\
\hline 93 & 3.393 & 365.38 & $\mathrm{f}=0.0170$ & 192 & 4.0445 & 306.55 & $\mathrm{f}=0.0055$ \\
\hline 94 & 3.396 & 365.14 & $\mathrm{f}=0.0065$ & 193 & 4.0475 & 306.33 & $\mathrm{f}=0.0061$ \\
\hline 95 & 3.398 & 364.84 & $\mathrm{f}=0.0034$ & 194 & 4.0635 & 305.11 & $\mathrm{f}=0.0017$ \\
\hline 96 & 3.413 & 363.31 & $\mathrm{f}=0.0088$ & 195 & 4.0678 & 304.79 & $\mathrm{f}=0.0120$ \\
\hline 97 & 3.417 & 362.85 & $\mathrm{f}=0.0181$ & 196 & 4.0729 & 304.41 & $\mathrm{f}=0.0049$ \\
\hline 98 & 3.423 & 362.21 & $\mathrm{f}=0.0037$ & 197 & 4.0816 & 303.77 & $\mathrm{f}=0.0181$ \\
\hline 99 & 3.425 & 361.99 & $\mathrm{f}=0.0615$ & 198 & 4.0845 & 303.55 & $\mathrm{f}=0.0097$ \\
\hline 100 & 3.434 & 361.01 & $\mathrm{f}=0.0007$ & 199 & 4.1 & 302.4 & $\mathrm{f}=0.0047$ \\
\hline 101 & 3.45 & 359.43 & $\mathrm{f}=0.0038$ & 200 & 4.1054 & 302.01 & $\mathrm{f}=0.0129$ \\
\hline
\end{tabular}

Table S8. Excited states information of porphyrin octant-thiophenes fullerene compound

\begin{tabular}{cccccccc}
\hline $\begin{array}{c}\text { Excited } \\
\text { States }\end{array}$ & $\begin{array}{c}\text { Excitation } \\
\text { Energy } \\
{[\mathbf{e V}]}\end{array}$ & $\begin{array}{c}\text { Absorption } \\
\text { Wavelength } \\
{[\mathbf{n m}]}\end{array}$ & $\begin{array}{c}\text { Oscillator } \\
\text { Strength }\end{array}$ & $\begin{array}{c}\text { Excited } \\
\text { States }\end{array}$ & $\begin{array}{c}\text { Excitation } \\
\text { Energy } \\
{[\mathbf{e V}]}\end{array}$ & $\begin{array}{c}\text { Absorption } \\
\text { Wavelength } \\
{[\mathbf{n m}]}\end{array}$ & $\begin{array}{c}\text { Oscillator } \\
\text { Strength }\end{array}$ \\
\hline $\mathbf{1}$ & 1.5917 & 778.96 & $\mathrm{f}=0.0114$ & $\mathbf{1 0 1}$ & 4.6213 & 268.29 & $\mathrm{f}=0.0213$ \\
\hline $\mathbf{2}$ & 2.0641 & 600.68 & $\mathrm{f}=0.0686$ & $\mathbf{1 0 2}$ & 4.6249 & 268.08 & $\mathrm{f}=0.0015$ \\
\hline $\mathbf{3}$ & 2.1807 & 568.55 & $\mathrm{f}=0.0093$ & $\mathbf{1 0 3}$ & 4.6298 & 267.8 & $\mathrm{f}=0.0194$ \\
\hline
\end{tabular}




\begin{tabular}{|c|c|c|c|c|c|c|c|}
\hline 4 & 2.2621 & 548.1 & $\mathrm{f}=0.2156$ & 104 & 4.6523 & 266.5 & $\mathrm{f}=0.0085$ \\
\hline 5 & 2.2697 & 546.25 & $\mathrm{f}=0.0070$ & 105 & 4.6602 & 266.05 & $\mathrm{f}=0.0021$ \\
\hline 6 & 2.3227 & 533.8 & $\mathrm{f}=0.0034$ & 106 & 4.6659 & 265.72 & $\mathrm{f}=0.0469$ \\
\hline 7 & 2.3257 & 533.1 & $\mathrm{f}=0.0041$ & 107 & 4.6726 & 265.34 & $\mathrm{f}=0.0022$ \\
\hline 8 & 2.449 & 506.26 & $\mathrm{f}=0.2639$ & 108 & 4.691 & 264.3 & $f=0.0068$ \\
\hline 9 & 2.4585 & 504.32 & $\mathrm{f}=0.0278$ & 109 & 4.6962 & 264.01 & $f=0.0056$ \\
\hline 10 & 2.552 & 485.84 & $\mathrm{f}=0.0182$ & 110 & 4.7054 & 263.49 & $f=0.0491$ \\
\hline 11 & 2.5616 & 484.01 & $f=3.6280$ & 111 & 4.7066 & 263.43 & $f=0.0426$ \\
\hline 12 & 2.6543 & 467.12 & $\mathrm{f}=0.0003$ & 112 & 4.7147 & 262.97 & $\mathrm{f}=0.0084$ \\
\hline 13 & 2.7049 & 458.36 & $\mathrm{f}=0.0003$ & 113 & 4.7255 & 262.37 & $\mathrm{f}=0.0039$ \\
\hline 14 & 2.8179 & 439.99 & $\mathrm{f}=0.0004$ & 114 & 4.7308 & 262.08 & $f=0.0002$ \\
\hline 15 & 2.8566 & 434.03 & $\mathrm{f}=0.0006$ & 115 & 4.7342 & 261.89 & $f=0.0086$ \\
\hline 16 & 2.9214 & 424.39 & $\mathrm{f}=0.0018$ & 116 & 4.743 & 261.4 & $\mathrm{f}=0.0024$ \\
\hline 17 & 2.9274 & 423.53 & $\mathrm{f}=0.0054$ & 117 & 4.7436 & 261.37 & $\mathrm{f}=0.0000$ \\
\hline 18 & 2.9711 & 417.3 & $\mathrm{f}=0.0100$ & 118 & 4.7545 & 260.77 & $\mathrm{f}=0.0179$ \\
\hline 19 & 3.0276 & 409.51 & $\mathrm{f}=0.0465$ & 119 & 4.7678 & 260.05 & $\mathrm{f}=0.0014$ \\
\hline 20 & 3.0412 & 407.69 & $\mathrm{f}=0.2019$ & 120 & 4.7837 & 259.18 & $\mathrm{f}=0.0420$ \\
\hline 21 & 3.0859 & 401.78 & $\mathrm{f}=0.0109$ & 121 & 4.785 & 259.11 & $\mathrm{f}=0.0107$ \\
\hline 22 & 3.1728 & 390.78 & $\mathrm{f}=0.0008$ & 122 & 4.7914 & 258.77 & $f=0.0743$ \\
\hline 23 & 3.2 & 387.45 & $\mathrm{f}=0.0025$ & 123 & 4.8022 & 258.18 & $\mathrm{f}=0.0058$ \\
\hline 24 & 3.2105 & 386.19 & $\mathrm{f}=0.0083$ & 124 & 4.8147 & 257.51 & $f=0.0096$ \\
\hline 25 & 3.2381 & 382.89 & $\mathrm{f}=0.0017$ & 125 & 4.8196 & 257.25 & $\mathrm{f}=0.0172$ \\
\hline 26 & 3.3199 & 373.45 & $f=0.6415$ & 126 & 4.8222 & 257.11 & $f=0.0019$ \\
\hline 27 & 3.3278 & 372.57 & $\mathrm{f}=0.8423$ & 127 & 4.8257 & 256.93 & $f=0.0775$ \\
\hline 28 & 3.3685 & 368.07 & $\mathrm{f}=0.0048$ & 128 & 4.8526 & 255.5 & $\mathrm{f}=0.0065$ \\
\hline 29 & 3.3943 & 365.27 & $\mathrm{f}=0.0005$ & 129 & 4.8593 & 255.15 & $\mathrm{f}=0.0139$ \\
\hline 30 & 3.4163 & 362.92 & $\mathrm{f}=0.0005$ & 130 & 4.8616 & 255.03 & $\mathrm{f}=0.0052$ \\
\hline 31 & 3.4217 & 362.35 & $\mathrm{f}=0.0000$ & 131 & 4.8714 & 254.51 & $\mathrm{f}=0.0046$ \\
\hline 32 & 3.4264 & 361.85 & $\mathrm{f}=0.0001$ & 132 & 4.874 & 254.38 & $\mathrm{f}=0.0280$ \\
\hline 33 & 3.4552 & 358.83 & $\mathrm{f}=0.0131$ & 133 & 4.8891 & 253.59 & $\mathrm{f}=0.0418$ \\
\hline 34 & 3.475 & 356.79 & $\mathrm{f}=0.0306$ & 134 & 4.8942 & 253.33 & $f=0.0038$ \\
\hline 35 & 3.4769 & 356.59 & $\mathrm{f}=0.6981$ & 135 & 4.9068 & 252.68 & $\mathrm{f}=0.0091$ \\
\hline 36 & 3.4969 & 354.55 & $\mathrm{f}=0.0030$ & 136 & 4.9125 & 252.39 & $\mathrm{f}=0.0415$ \\
\hline 37 & 3.556 & 348.66 & $\mathrm{f}=0.0229$ & 137 & 4.9244 & 251.78 & $\mathrm{f}=0.0008$ \\
\hline 38 & 3.5805 & 346.27 & $f=0.1888$ & 138 & 4.9283 & 251.57 & $\mathrm{f}=0.0040$ \\
\hline 39 & 3.6173 & 342.76 & $\mathrm{f}=0.0006$ & 139 & 4.9419 & 250.88 & $\mathrm{f}=0.0329$ \\
\hline 40 & 3.631 & 341.46 & $\mathrm{f}=0.0062$ & 140 & 4.9571 & 250.12 & $\mathrm{f}=0.0039$ \\
\hline 41 & 3.6337 & 341.21 & $\mathrm{f}=0.0006$ & 141 & 4.9647 & 249.73 & $\mathrm{f}=0.0140$ \\
\hline 42 & 3.6497 & 339.71 & $\mathrm{f}=0.1055$ & 142 & 4.968 & 249.57 & $\mathrm{f}=0.0046$ \\
\hline 43 & 3.6856 & 336.4 & $\mathrm{f}=0.0035$ & 143 & 4.9763 & 249.15 & $\mathrm{f}=0.0033$ \\
\hline 44 & 3.7112 & 334.08 & $\mathrm{f}=0.0245$ & 144 & 4.9853 & 248.7 & $\mathrm{f}=0.0064$ \\
\hline 45 & 3.7245 & 332.89 & $\mathrm{f}=0.0016$ & 145 & 4.9913 & 248.4 & $\mathrm{f}=0.0054$ \\
\hline 46 & 3.7504 & 330.59 & $\mathrm{f}=0.0105$ & 146 & 5.0022 & 247.86 & $\mathrm{f}=0.0078$ \\
\hline
\end{tabular}




\begin{tabular}{|c|c|c|c|c|c|c|c|}
\hline 47 & 3.7634 & 329.45 & $\mathrm{f}=0.0110$ & 147 & 5.0168 & 247.14 & $\mathrm{f}=0.0034$ \\
\hline 48 & 3.8125 & 325.21 & $f=0.0068$ & 148 & 5.0192 & 247.02 & $\mathrm{f}=0.0025$ \\
\hline 49 & 3.8184 & 324.7 & $\mathrm{f}=0.0000$ & 149 & 5.0259 & 246.69 & $\mathrm{f}=0.0119$ \\
\hline 50 & 3.8245 & 324.18 & $\mathrm{f}=0.0090$ & 150 & 5.0336 & 246.31 & $f=0.0064$ \\
\hline 51 & 3.8525 & 321.83 & $\mathrm{f}=0.0122$ & 151 & 5.0338 & 246.3 & $\mathrm{f}=0.0038$ \\
\hline 52 & 3.8732 & 320.11 & $\mathrm{f}=0.0032$ & 152 & 5.0377 & 246.11 & $\mathrm{f}=0.0021$ \\
\hline 53 & 3.8775 & 319.75 & $\mathrm{f}=0.0103$ & 153 & 5.0419 & 245.91 & $f=0.0018$ \\
\hline 54 & 3.9167 & 316.55 & $\mathrm{f}=0.0052$ & 154 & 5.0551 & 245.27 & $f=0.0160$ \\
\hline 55 & 3.9412 & 314.59 & $\mathrm{f}=0.0249$ & 155 & 5.0689 & 244.6 & $\mathrm{f}=0.0074$ \\
\hline 56 & 3.9551 & 313.48 & $\mathrm{f}=0.0093$ & 156 & 5.0722 & 244.44 & $\mathrm{f}=0.0093$ \\
\hline 57 & 3.9561 & 313.4 & $f=0.0699$ & 157 & 5.0764 & 244.24 & $f=0.0074$ \\
\hline 58 & 3.9937 & 310.45 & $f=0.1071$ & 158 & 5.0791 & 244.11 & $f=0.0089$ \\
\hline 59 & 4.0383 & 307.02 & $f=0.0016$ & 159 & 5.0825 & 243.94 & $f=0.0031$ \\
\hline 60 & 4.0563 & 305.66 & $\mathrm{f}=0.3224$ & 160 & 5.0919 & 243.49 & $\mathrm{f}=0.0022$ \\
\hline 61 & 4.061 & 305.3 & $\mathrm{f}=0.0000$ & 161 & 5.0971 & 243.24 & $\mathrm{f}=0.0057$ \\
\hline 62 & 4.0631 & 305.15 & $\mathrm{f}=0.0285$ & 162 & 5.0993 & 243.14 & $\mathrm{f}=0.0134$ \\
\hline 63 & 4.0699 & 304.64 & $\mathrm{f}=0.0731$ & 163 & 5.1061 & 242.82 & $\mathrm{f}=0.0071$ \\
\hline 64 & 4.0762 & 304.16 & $\mathrm{f}=0.0527$ & 164 & 5.1125 & 242.51 & $f=0.0009$ \\
\hline 65 & 4.1242 & 300.63 & $\mathrm{f}=0.0091$ & 165 & 5.124 & 241.97 & $f=0.0089$ \\
\hline 66 & 4.1581 & 298.18 & $\mathrm{f}=0.0092$ & 166 & 5.1264 & 241.85 & $f=0.0699$ \\
\hline 67 & 4.1646 & 297.71 & $f=0.0065$ & 167 & 5.1317 & 241.6 & $\mathrm{f}=0.0126$ \\
\hline 68 & 4.1741 & 297.03 & $f=0.0654$ & 168 & 5.1337 & 241.51 & $\mathrm{f}=0.0044$ \\
\hline 69 & 4.1851 & 296.25 & $\mathrm{f}=0.0056$ & 169 & 5.137 & 241.36 & $\mathrm{f}=0.0080$ \\
\hline 70 & 4.2168 & 294.02 & $\mathrm{f}=0.0378$ & 170 & 5.1405 & 241.19 & $\mathrm{f}=0.0270$ \\
\hline 71 & 4.2189 & 293.88 & $\mathrm{f}=0.0019$ & 171 & 5.1451 & 240.98 & $\mathrm{f}=0.0060$ \\
\hline 72 & 4.2439 & 292.14 & $\mathrm{f}=0.0064$ & 172 & 5.1556 & 240.49 & $\mathrm{f}=0.0001$ \\
\hline 73 & 4.2464 & 291.97 & $\mathrm{f}=0.0125$ & 173 & 5.1592 & 240.32 & $\mathrm{f}=0.0026$ \\
\hline 74 & 4.265 & 290.7 & $\mathrm{f}=0.0036$ & 174 & 5.1644 & 240.07 & $\mathrm{f}=0.0142$ \\
\hline 75 & 4.2808 & 289.63 & $\mathrm{f}=0.0023$ & 175 & 5.1652 & 240.04 & $\mathrm{f}=0.0992$ \\
\hline 76 & 4.2933 & 288.79 & $\mathrm{f}=0.0026$ & 176 & 5.1724 & 239.7 & $\mathrm{f}=0.0171$ \\
\hline 77 & 4.3144 & 287.37 & $\mathrm{f}=0.0727$ & 177 & 5.1786 & 239.42 & $f=0.0035$ \\
\hline 78 & 4.3292 & 286.39 & $\mathrm{f}=0.0071$ & 178 & 5.1797 & 239.37 & $\mathrm{f}=0.0029$ \\
\hline 79 & 4.3323 & 286.19 & $\mathrm{f}=0.0084$ & 179 & 5.1822 & 239.25 & $\mathrm{f}=0.0038$ \\
\hline 80 & 4.3549 & 284.7 & $\mathrm{f}=0.0184$ & 180 & 5.1893 & 238.92 & $\mathrm{f}=0.0011$ \\
\hline 81 & 4.3608 & 284.32 & $\mathrm{f}=0.0052$ & 181 & 5.1923 & 238.78 & $\mathrm{f}=0.0038$ \\
\hline 82 & 4.3772 & 283.25 & $\mathrm{f}=0.0008$ & 182 & 5.1975 & 238.54 & $\mathrm{f}=0.0371$ \\
\hline 83 & 4.3937 & 282.19 & $\mathrm{f}=0.0402$ & 183 & 5.2038 & 238.26 & $\mathrm{f}=0.0325$ \\
\hline 84 & 4.3977 & 281.93 & $\mathrm{f}=0.0088$ & 184 & 5.2081 & 238.06 & $\mathrm{f}=0.0236$ \\
\hline 85 & 4.4244 & 280.23 & $\mathrm{f}=0.0124$ & 185 & 5.2204 & 237.5 & $\mathrm{f}=0.0004$ \\
\hline 86 & 4.4536 & 278.39 & $\mathrm{f}=0.0049$ & 186 & 5.2251 & 237.29 & $\mathrm{f}=0.0197$ \\
\hline 87 & 4.4638 & 277.75 & $\mathrm{f}=0.0229$ & 187 & 5.2299 & 237.07 & $\mathrm{f}=0.0089$ \\
\hline 88 & 4.4686 & 277.46 & $\mathrm{f}=0.1481$ & 188 & 5.2323 & 236.96 & $\mathrm{f}=0.0135$ \\
\hline 89 & 4.4918 & 276.02 & $\mathrm{f}=0.0371$ & 189 & 5.2358 & 236.8 & $\mathrm{f}=0.0034$ \\
\hline
\end{tabular}




\begin{tabular}{cccccccc}
\hline $\mathbf{9 0}$ & 4.4951 & 275.82 & $\mathrm{f}=0.0226$ & $\mathbf{1 9 0}$ & 5.2452 & 236.38 & $\mathrm{f}=0.0249$ \\
\hline $\mathbf{9 1}$ & 4.4998 & 275.53 & $\mathrm{f}=0.0097$ & $\mathbf{1 9 1}$ & 5.2515 & 236.09 & $\mathrm{f}=0.0327$ \\
\hline $\mathbf{9 2}$ & 4.5078 & 275.04 & $\mathrm{f}=0.0040$ & $\mathbf{1 9 2}$ & 5.2635 & 235.55 & $\mathrm{f}=0.0050$ \\
\hline $\mathbf{9 3}$ & 4.5152 & 274.59 & $\mathrm{f}=0.0045$ & $\mathbf{1 9 3}$ & 5.2657 & 235.46 & $\mathrm{f}=0.0291$ \\
\hline $\mathbf{9 4}$ & 4.5258 & 273.95 & $\mathrm{f}=0.0011$ & $\mathbf{1 9 4}$ & 5.2722 & 235.17 & $\mathrm{f}=0.0058$ \\
\hline $\mathbf{9 5}$ & 4.5357 & 273.35 & $\mathrm{f}=0.0123$ & $\mathbf{1 9 5}$ & 5.2732 & 235.12 & $\mathrm{f}=0.0132$ \\
\hline $\mathbf{9 6}$ & 4.54 & 273.09 & $\mathrm{f}=0.0089$ & $\mathbf{1 9 6}$ & 5.2767 & 234.96 & $\mathrm{f}=0.0211$ \\
\hline $\mathbf{9 7}$ & 4.5552 & 272.18 & $\mathrm{f}=0.0089$ & $\mathbf{1 9 7}$ & 5.2786 & 234.88 & $\mathrm{f}=0.0119$ \\
\hline $\mathbf{9 8}$ & 4.5746 & 271.03 & $\mathrm{f}=0.0065$ & $\mathbf{1 9 8}$ & 5.2793 & 234.85 & $\mathrm{f}=0.0295$ \\
\hline $\mathbf{9 9}$ & 4.5762 & 270.93 & $\mathrm{f}=0.0106$ & $\mathbf{1 9 9}$ & 5.2844 & 234.62 & $\mathrm{f}=0.0346$ \\
\hline $\mathbf{1 0 0}$ & 4.6199 & 268.37 & $\mathrm{f}=0.0045$ & $\mathbf{2 0 0}$ & 5.2873 & 234.5 & $\mathrm{f}=0.0008$ \\
\hline
\end{tabular}

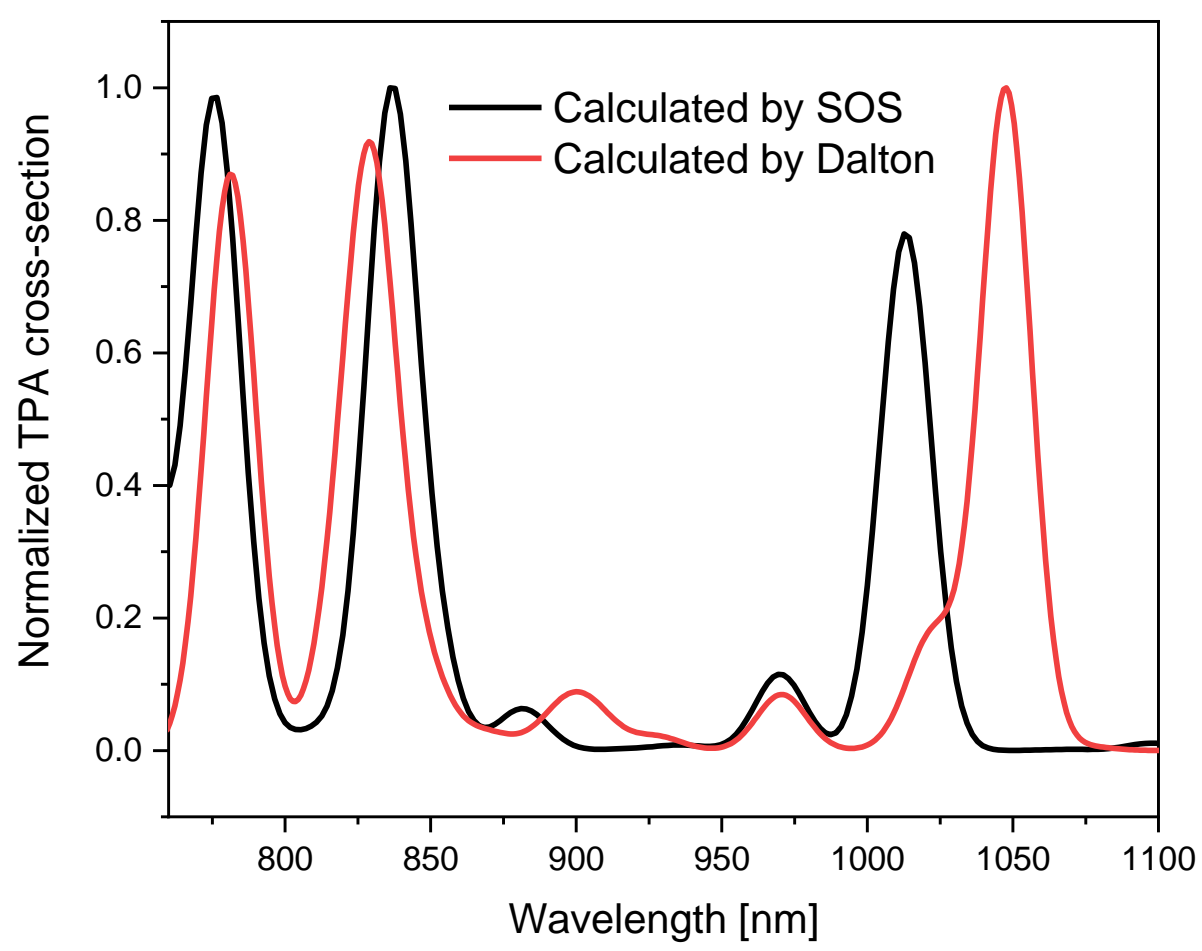

Figure S2. The TPA spectra of porphyrin octant-thiophenes-fullerene calculated by SOS method and Dalton-2016.

\section{Complete references}

[28] Frisch, M. J. ; Trucks, G. W. ; Schlegel, H. B. ; Scuseria, G. E. ; Robb, M. A. ;

Cheeseman, J. R. ;Scalmani, G. ;Barone, V. ;Petersson, G. A. ;Nakatsuji, H. ;Li, X. ;

Caricato, M. ; Marenich, A. V. ; Bloino, J. ; Janesko, B. G. ; Gomperts, R. ; Mennucci, 
B. ; Hratchian, H. P. ; Ortiz, J. V. ; Izmaylov, A. F. ; Sonnenberg, J. L. ; Williams ; Ding, F. ;Lipparini, F. ;Egidi, F. ;Goings, J. ;Peng, B. ;Petrone, A. ;Henderson, T. ; Ranasinghe, D. ;Zakrzewski, V. G. ;Gao, J. ;Rega, N. ;Zheng, G. ;Liang, W. ;Hada, M. ; Ehara, M. ; Toyota, K. ; Fukuda, R. ; Hasegawa, J. ; Ishida, M. ; Nakajima, T. ; Honda, Y. ; Kitao, O. ; Nakai, H. ; Vreven, T. ; Throssell, K. ; Montgomery Jr., J. A. ; Peralta, J. E. ; Ogliaro, F. ; Bearpark, M. J. ; Heyd, J. J. ; Brothers, E. N. ; Kudin, K. N. ;Staroverov, V. N. ;Keith, T. A. ;Kobayashi, R. ;Normand, J. ;Raghavachari, K. ; Rendell, A. P. ; Burant, J. C. ; Iyengar, S. S. ; Tomasi, J. ; Cossi, M. ; Millam, J. M. ; Klene, M. ;Adamo, C. ;Cammi, R. ;Ochterski, J. W. ;Martin, R. L. ;Morokuma, K. ; Farkas, O. ; Foresman, J. B. ; Fox, D. J.; Gaussian 09, 2009. Wallingford CT.

[36] Aidas, K. ; Angeli, C. ; Bak, K. L. ; Bakken, V. ; Bast, R. ; Boman, L. ; Christiansen, O. ;Cimiraglia, R. ;Coriani, S. ;Dahle, P. ;Dalskov, E. K. ;Ekström, U. ; Enevoldsen, T. ;Eriksen, J. J. ;Ettenhuber, P. ;Fernández, B. ;Ferrighi, L. ;Fliegl, H. ; Frediani, L. ; Hald, K. ; Halkier, A. ; Hättig, C. ; Heiberg, H. ; Helgaker, T. ; Hennum, A. C. ;Hettema, H. ;Hjertenæs, E. ;Høst, S. ;Høyvik, I.-M. ;Iozzi, M. F. ;Jansík, B. ; Jensen, H. J. A. ; Jonsson, D. ; Jørgensen, P. ; Kauczor, J. ; Kirpekar, S. ; Kjærgaard, T. ; Klopper, W. ; Knecht, S. ; Kobayashi, R. ; Koch, H. ; Kongsted, J. ; Krapp, A. ; Kristensen, K. ; Ligabue, A. ; Lutnæs, O. B. ; Melo, J. I. ; Mikkelsen, K. V. ; Myhre, R. H. ;Neiss, C. ;Nielsen, C. B. ;Norman, P. ;Olsen, J. ;Olsen, J. M. H. ;Osted, A. ; Packer, M. J. ; Pawlowski, F. ;Pedersen, T. B. ;Provasi, P. F. ; Reine, S. ; Rinkevicius, Z. ; Ruden, T. A. ; Ruud, K. ; Rybkin, V. V. ; Sałek, P. ; Samson, C. C. M. ; de Merás, A. S. ;Saue, T. ;Sauer, S. P. A. ;Schimmelpfennig, B. ;Sneskov, K. ;Steindal, A. H. ; 
Sylvester-Hvid, K. O. ; Taylor, P. R. ; Teale, A. M. ; Tellgren, E. I. ; Tew, D. P. ; Thorvaldsen, A. J. ; Thøgersen, L. ; Vahtras, O. ; Watson, M. A. ; Wilson, D. J. D. ; Ziolkowski, M. ;Ågren, H.; The Dalton Quantum Chemistry Program System, WIREs. Comput. Mol. Sci., 2014, 4, 269-284. 